\title{
Urban Near-surface Seismic Monitoring using Distributed Acoustic Sensing
}

Gang Fang ${ }^{1,2}$, Yunyue Elita $\mathrm{Li}^{1}$, Yumin Zhao $^{1}$, and Eileen R. Martin ${ }^{3}$

\footnotetext{
${ }^{1}$ Department of Civil and Environmental Engineering, National University of Singapore, Singapore ${ }^{2}$ Key Laboratory of Marine Hydrocarbon Resources and Environmental Geology, Ministry of Land and Resources, Qingdao Institute of Marine Geology, Qingdao, 266071, China

${ }^{3}$ Department of Mathematics, Program in Computational Modeling and Data Analytics, Virginia Tech, Virginia, USA
}

\section{Key Points:}

- Using the Stanford DAS array, we demonstrate the reliability of urban DAS recordings when deployed in existing infrastructures.

- Short DAS recordings of far-field quarry blasts show sensitivity to the changes in near-surface velocity within the boundaries of the array.

- DAS can play an important role in real time, high resolution, and long term urban monitoring applications.

Corresponding author: Yunyue Elita Li, elita.li@nus.edu.sg 


\begin{abstract}
Urban subsurface monitoring requires high temporal-spatial resolution, low maintenance cost, and minimal intrusion to nearby life. Distributed acoustic sensing (DAS), in contrast to conventional station-based sensing technology, has the potential to provide a passive seismic solution to urban monitoring requirements. Based on data recorded by the Stanford Fiber Optic Seismic Observatory, we demonstrate that near-surface velocity changes induced by the excavation of a basement construction can be monitored using existing fiber optic infrastructure in a noisy urban environment. To achieve the satisfactory results, careful signal processing comprising of noise removal and source signature normalization are applied to raw DAS recordings. Repeated blast signals from quarry sites provide free, unidirectional, and near-impulsive sources for periodic urban seismic monitoring, which are essential for increasing the temporal resolution of passive seismic methods. Our study suggests that DAS will likely play an important role in urban subsurface monitoring.
\end{abstract}

\title{
1 Plain Language Summary
}

Seismic monitoring can provide crucial information about near-surface changes due to natural or manmade activities. However, the high cost and the "after-effect" nature of conventional station-based monitoring methods limit their application in urban environments where near real-time and meter-scale resolution are required. Distributed acoustic sensing (DAS) has the potential to achieve all requirements utilizing existing communication infrastructure. Using Stanford Fiber Optic Seismic Observatory, we demonstrate that its recordings of quarry blasts $13.3 \mathrm{~km}$ away carry important subsurface velocity information within the footprint of the array. These short bursts of quarry blast signals provide us free, unidirectional, and repetitive sources that sample the urban subsurface at an interval frequent enough for monitoring. We observe large velocity decrease from the recordings close to the excavation site. Our study suggests that telecommunications fiber repurposed for DAS will potentially play an important role in many urban subsurface monitoring applications.

\section{Introduction}

Characterizing and monitoring changes in the top tens of meters of the Earth's subsurface will play a significant role in satisfying the increasing need for urban sustainability and resilience (Díaz et al., 2017). Near-surface changes due to natural or man-made events may lead to hazards including ground subsidence (Tran \& Sperry, 2018), sinkholes (Dahm et al., 2011; Gutiérrez et al., 2014), and landslides (Renalier et al., 2010; Schenato et al., 2017), which may result in direct casualties and damages to existing infrastructure (Douglas, 2004). Many such subsurface changes manifest themselves as temporal variations in geophysical properties (such as velocity, attenuation, electric conductivity, gravity, etc.) before catastrophic hazards occur, which can be monitored and predicted by geophysical prospecting.

Compared to conventional geophysical exploration for resources, near-surface monitoring in urban environments has unique acquisition requirements including high spatial resolution towards meter-scale, high temporal resolution towards real-time data collection and daily warning, low maintenance cost for long term monitoring and minimal intrusion to urban life. These requirements are met by a passive system enabled by DAS that we present in this paper. DAS arrays can measure strain along kilometers of optical fiber, producing large datasets with kilohertz time sampling and at sub-meter channel spacing (Parker et al., 2014). Over the past decade, DAS has been a rapidly evolving technology for downhole recording in oil and gas reservoirs (Willis et al., 2016). Recent success of DAS applications using existing telecommunication infrastructures (Jousset et al., 2018; Yu et al., 2019; Ajo-Franklin et al., 2019) demonstrates its cost-effectiveness 
in deployment and maintenance. However, these experiments are conducted for applications in earthquake seismology in remote areas where anthropogenic noise is rare and desired signals are clearly visible above the random noise in DAS measurements.

Studies using DAS arrays deployed in urban environments have reported that nearsurface velocities can be estimated with ambient noise recorded by DAS over month-long periods (Dou et al., 2017; Martin et al., 2018; Spica et al., 2019). Averaging over long observation times is needed to suppress strong near-field anthropogenic noise, but severely limits the temporal resolution of passive seismic monitoring with DAS. Here we present a case study from the Stanford Fiber Optic Seismic Observatory where we take advantage of far-field anthropogenic activities - quarry blasts - to monitor the near-field anthropogenic activity - excavation. Weekly quarry blasts can be used to sample the subsurface with sufficient energy at intervals relevant to urban monitoring. We perform careful signal processing to reduce the effect of strong nearby noise and the variability in the blast sources. We demonstrate that with 100 seconds of DAS recordings after quarry blasts, near-surface velocity changes caused by construction of a basement within the array can be observed.

\section{Data and Signal Processing}

\subsection{Stanford Fiber Optic Seismic Observatory data acquisition}

The Stanford Fiber Optic Seismic Observatory (also called Stanford DAS Array) (Biondi et al., 2017) is one of the first DAS arrays to use existing telecommunication infrastructure, and is the longest-running ultra-dense urban seismic study in the world. In this experiment, 2.5 kilometers of fiber-optic cable are deployed loosely in existing underground telecommunication conduits (typically $10-15 \mathrm{~cm}$ wide PVC pipes) under the Stanford University campus. Coupling between the fiber cable and the surrounding conduit relies only on gravity and friction. This experiment simulates DAS acquisition using dark fibers (the unused backup fiber-optic cables) that are commonly available in existing telecommunication systems. Figure 1a shows the layout of the DAS array, which records data at a $25 \mathrm{~Hz}$ Nyquist frequency with $8.16 \mathrm{~m}$ channel spacing and $7.14 \mathrm{~m}$ gauge length (Dean et al., 2017; Lindsey et al., 2017). Construction of a basement (labeled with "A" in Figure 1a) began by its excavation on 7 November 2016 .

\subsection{Quarry blasts data}

Lehigh Permanente Quarry is located $13.3 \mathrm{~km}$ away $29.9^{\circ}$ southeast of the DAS array (Figure 1b). Figures 1c and 1d show the DAS recordings on 12 October 201618 : $30: 16.9$ UTC and on 21 November $18: 56: 12.5$ UTC, after applying a bandpass filter from 0.25 to $2.5 \mathrm{~Hz}$. The origin of the time axis denotes the blasting time provided by analysis of the data recorded by a USGS seismometer at the Jasper Ridge seismic station (JRSC) that is managed by the Berkeley Digital Seismic Network. The near vertical events originate from the quarry blasts, whereas strong dipping events are the direct impact of traffic on the fiber and the horizontal events are construction noise. We observe polarity flips around the corners of each pair of orthogonal segments of the DAS array (Figures 1c, 1d and Movie S1 in supporting information), which are caused by the angular sensitivity of DAS strain measurements (Lindsey et al., 2017). Table S1 in supporting information lists the time and the magnitude of 10 quarry blast events used for further analysis.

In the subsequent signal processing section, we aim to extract subsurface information based on far-field quarry blasts while minimizing the influence of near-field anthropogenic noise. Figures 2a and $2 \mathrm{~b}$ zoom in on two blast signals after geometric polarity sign-correction (Biondi et al., 2017). Because of the strong surface wave energy originating from the quarry blast and anthropogenic noise (mainly traffic and construction noise) 


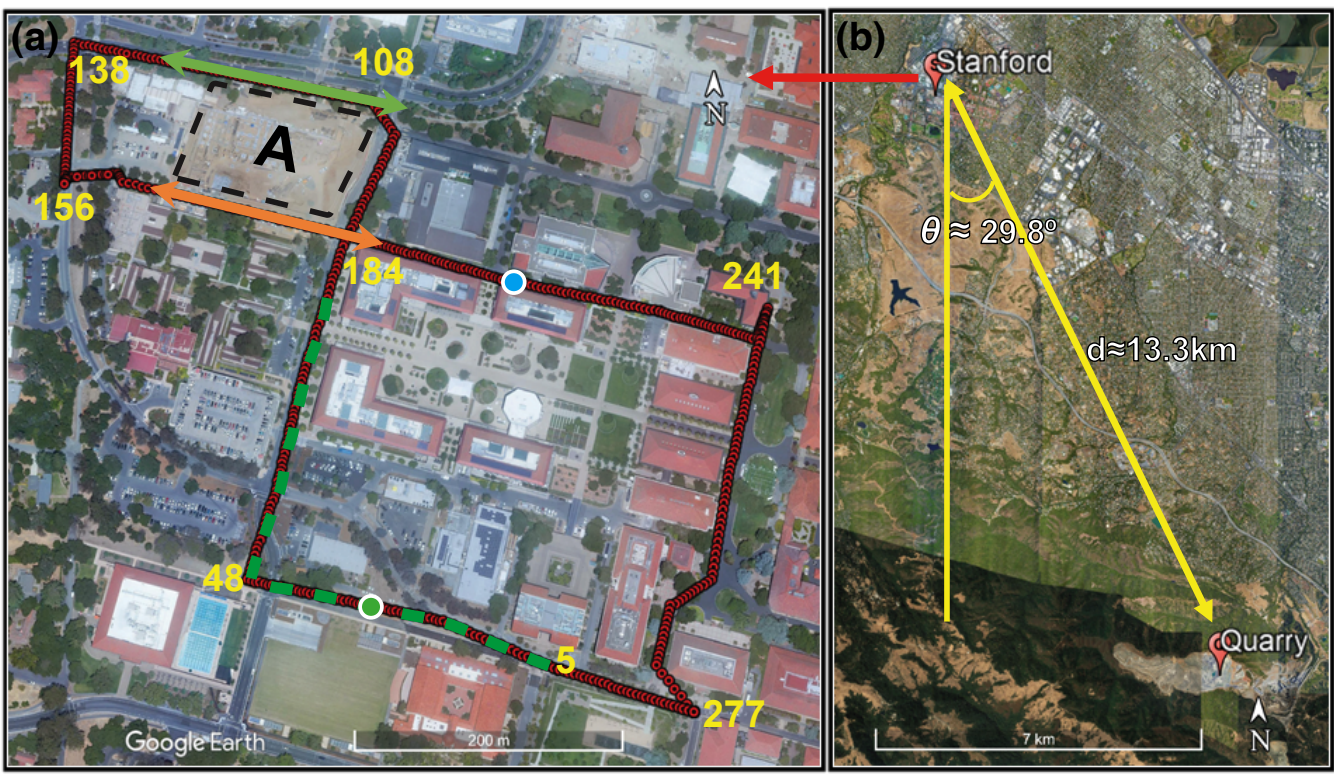

(c)

$2016 / 10 / 12$
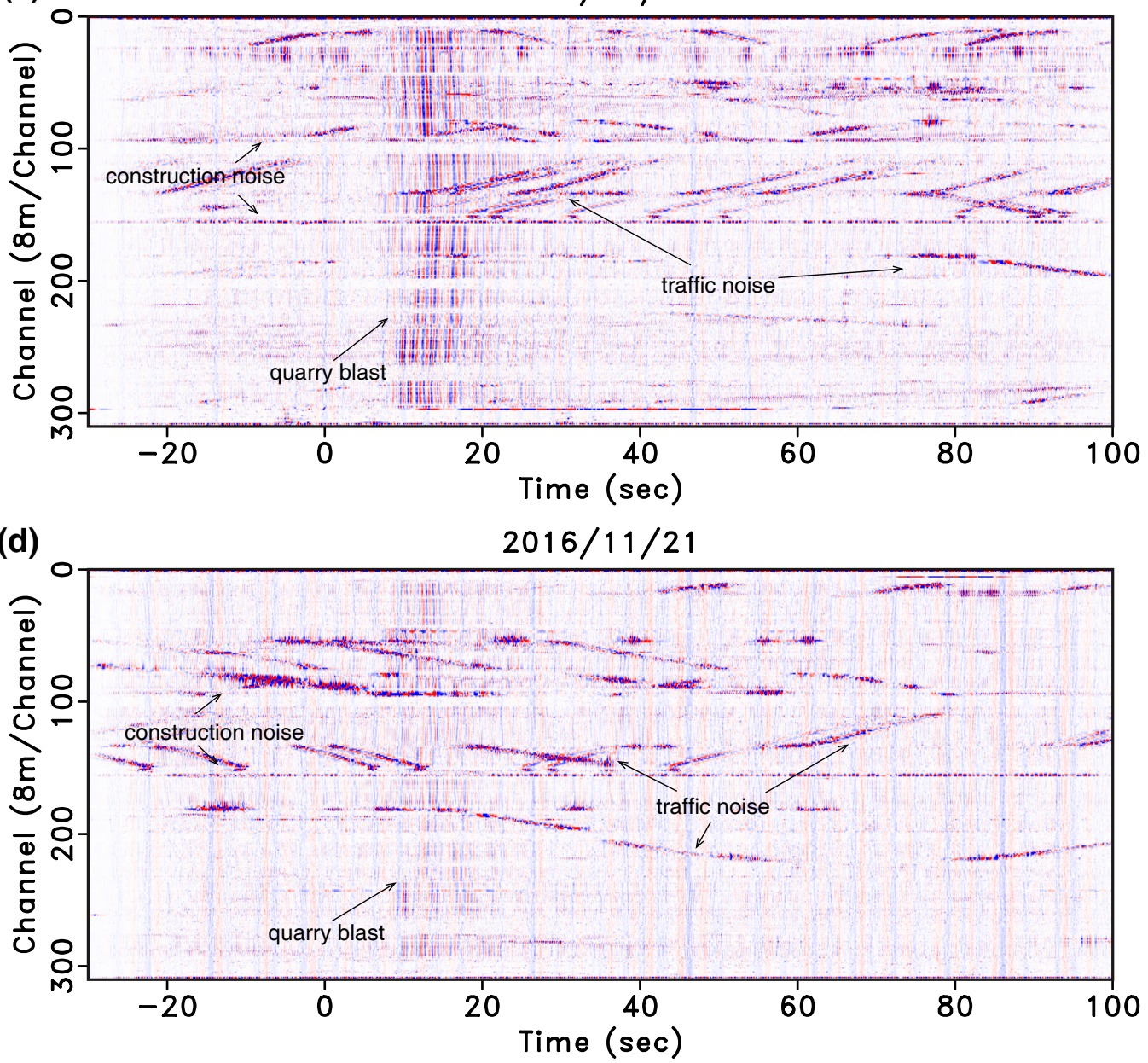

Figure 1. (a) Layout of the DAS array with the corner points labeled by the corresponding channel numbers. The green dashed line represents the segment of DAS recording used for beamforming calculation in Figure 2. The green and orange arrows represent the segment of DAS recording used in Figure 4. The green and blue dots are virtual sources used for seismic interferometry in Figures 4 and 5, respectively. Box A denotes the basement construction site. (b) Location of the quarry relative to the DAS array. (c) and (d) Bandpassed DAS recordings on 12 October 2016 and 21 November 2016, respectively. 
during the daytime, it is hard to identify any body wave in the records. Based on the facts that the quarry blasts events propagate through the DAS array in a non-perpendicular uniform direction and the arrival times of Rayleigh and Love waves are close, a combination of Rayleigh and Love waves are expected to be observed by the DAS array. The blast vibration events reach the south portion of the array (channel 5) almost $0.7 \mathrm{~s}$ earlier than they reach the north portion (channel 138). This time lag matches the relative distance and the average velocity between these two portions of the array, which is estimated in the next section. The blue lines overlaid on the profiles of Figures $2 \mathrm{a}$ and $2 \mathrm{~b}$ are the single-channel responses of channel 120 on two different days. Figures $2 \mathrm{c}$ and $2 \mathrm{~d}$ show their time-frequency spectrograms, respectively. Note that the main energy of the two quarry blasts events arrives at the DAS array around 14s after the explosion. Their dominant frequencies are approximately $1.2 \mathrm{~Hz}$.

Beamforming based on multiple signal classification (MUSIC) (Zhang et al., 2019) is applied on the southwest corner of the DAS array (indicated by green dashed line in Figure 1a) within a small time window of 10-15s (labeled with the red dashed box in Figures $2 \mathrm{a}$ and $2 \mathrm{~b}$ ). Figures $2 \mathrm{e}, 2 \mathrm{f}$ and movie $\mathrm{S} 2$ in supporting information show the beamforming results for different days' data. Their peaks roughly indicate the wavefield propagation direction and velocity, which support the assumption that the quarry blasts can be seen as unidirectional plane wave sources. Although the quarry blasts signals recorded at different times show certain similarity, their waveforms are complex and quite different as shown by the blue lines in Figures 2a and 2b. The reasons for this difference may lie in the randomness of explosive energy, excitation environment and the rugged earth surface where the source is excited. Urban noise further contaminates the signals.

\subsection{Signal processing}

We propose a data processing workflow to reduce the impact of urban noise and waveform differences. It starts with raw DAS records and results in cross-correlograms between virtual sources and other channels, which are used for velocity estimation. The workflow is the same for all quarry blasts.

Bandpass, FK and median filter: A Butterworth bandpass filter with cut-off frequencies from $0.25 \mathrm{~Hz}$ to $2.5 \mathrm{~Hz}$ is applied to all quarry blast data. A narrowband frequency-wavenumber(FK) filter is applied to remove the high frequency, large moveout events, which are primarily generated by direct traffic impact or the equipment on the construction site. Therefore, the parameters to control the FK filter are tuned daily according to noise on that day. A sliding 2-D median filter is used to remove spike noise for all the data.

Normalized cross-correlation: The quarry sets off each blast with a different source signature. We use normalized cross-correlation to eliminate the imprint of the source signature. Under the assumption of far-field plane-wave propagation and uniform receiver response, the signals $U$ recorded at receivers $A$ and $B$ in the frequency domain can be approximated by 1-D wave propagation as follows

$$
U\left(R_{A}, \omega\right)=S\left(R_{S}, \omega\right) e^{i k D i s\left(R_{A}, R_{S}\right)}
$$

$$
U\left(R_{B}, \omega\right)=S\left(R_{S}, \omega\right) e^{i k D i s\left(R_{B}, R_{S}\right)},
$$

where $S\left(R_{S}, \omega\right)$ is the source spectrum, $k$ is the wavenumber, $R_{A}$ and $R_{B}$ are locations of $\mathrm{A}$ and $\mathrm{B}, \operatorname{Dis}\left(R_{A}, R_{S}\right)$ are the distance between $R_{A}$ and $R_{S}$, respectively. The normalized cross-correlation operator is defined as

$$
C_{N}\left(R_{A}, R_{B}, \omega\right)=\frac{U\left(R_{A}, \omega\right) U^{*}\left(R_{B}, \omega\right)}{<<U\left(R_{B}, \omega\right) U^{*}\left(R_{B}, \omega\right)>>} \approx e^{i k D i s\left(R_{A}, R_{B}\right)},
$$



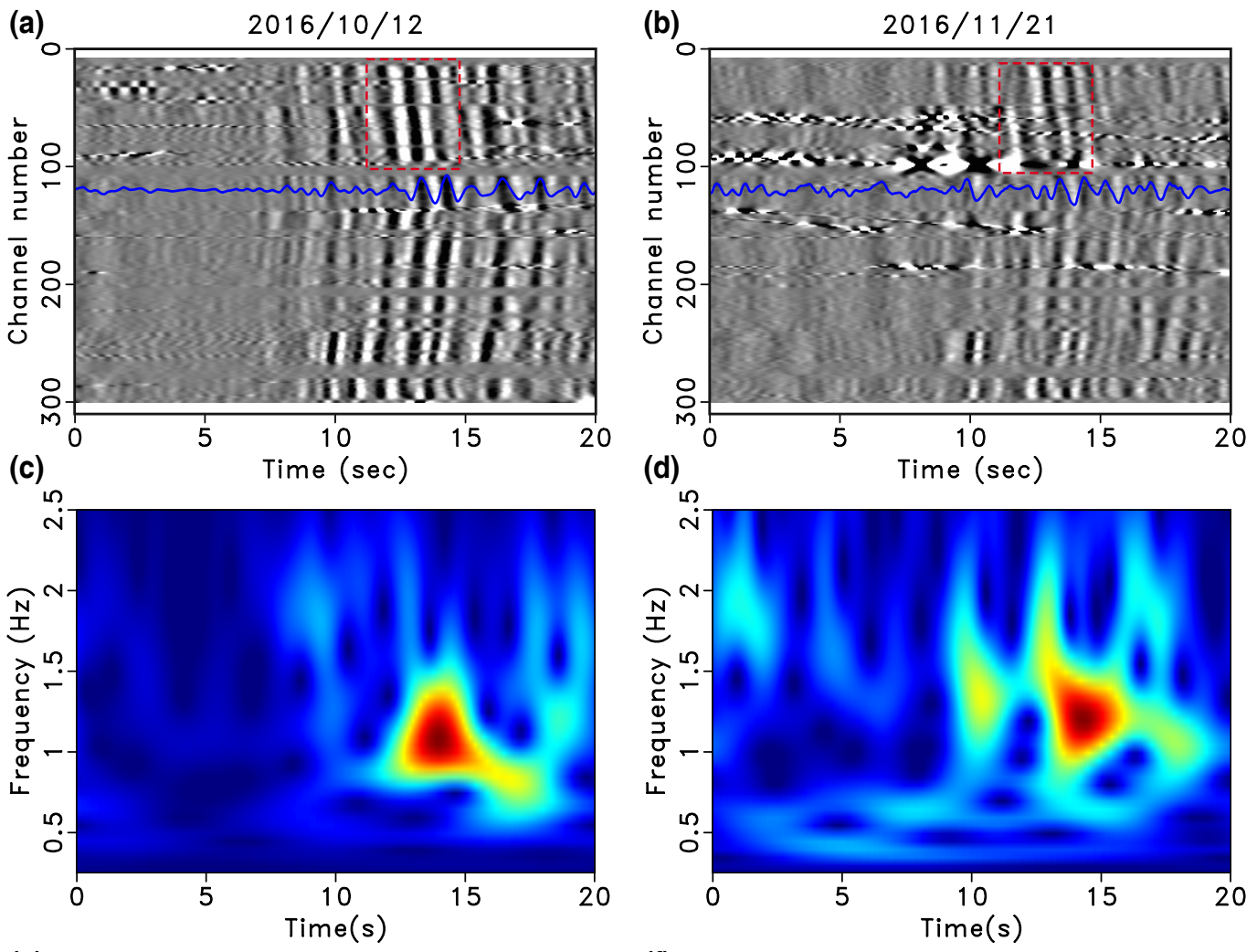

(e)

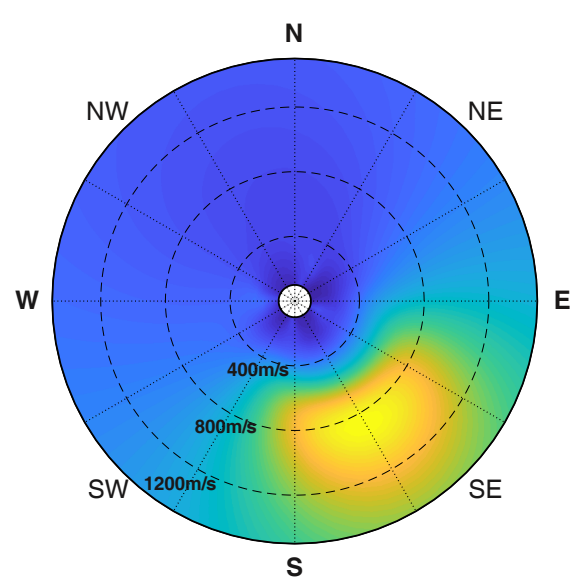

(f)

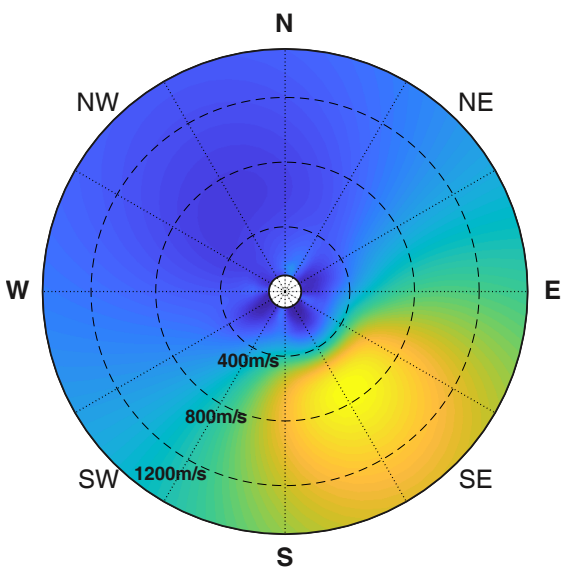

Figure 2. Quarry blasts DAS data on (a) 12 October 2016 and (b) 21 November 2016. Both plots (a) and (b) are after noise attenuation and polarity correction. Blue lines denote the signals at channel 120. Plots (c) and (d) compare the time frequency spectrograms of these two days data, which are calculated with the single channel shown with blue lines in plot (a) and (b), respectively. Plot (e) and (f) compare with the beamforming spectrum calculated with the data in the red dashed box in (a) and (b), whose peaks indicate the wavefield direction of propagation and its velocity. The channels used for beamforming are from 12-77, indicated by green dashed line in Figure 1a. 
where $<<\cdot>>$ is a Gaussian smoothing operator. Equation 3 is an implementation of deconvolution in the frequency domain, which can both remove the influence of the source wavelet and improve the data resolution. More details of data processing results can be found in supporting information Figures S1-S2.

\section{Results}

Using data recorded at channels away from the construction site, we first establish the baseline velocity of the site and demonstrate that DAS recordings, after urban noise removal, can provide reliable velocity estimates. We select channels $5-48$, use 5 channels near channel 5 as virtual sources, and calculate the normalized cross-correlograms. Figure 3a shows one of these normalized cross-correlograms on 12 October 2016, whose vertical axis represents the seismic time lag from virtual source to channels. The channels that are still contaminated by near-field noise after signal processing are omitted. Figure 3b shows the picked travel-time lag along the distance between virtual source and receiver, where the different colored dots denote the picks from different virtual sources. Figures $3 \mathrm{c}$ and $3 \mathrm{~d}$ are similar to Figures $3 \mathrm{a}$ and $3 \mathrm{~b}$ but computed on 21 November 2016. The surface seismic velocities are estimated by a least-squares linear regression of the picked travel times on each day. After correction for the propagation angles obtained from beamforming spectrums (as shown in Figures 2e and 2f), the measured velocities on 10 different days show small variations over 3 months (Table S1 in supporting information). The average velocity over 3 months is measured at $816 \mathrm{~m} / \mathrm{s}$ and their coefficient of variation is $3.2 \%$, with which the measurements at the construction site are benchmarked.

When we focus on the segment of the array closer to the construction site, the effects of excavation on velocity are observed. We select two segments of the DAS recordings surrounding the construction site, one on the south edge (channels 170-184), and the other on the north edge (channels 108-128). Figure 4 shows the normalized crosscorrelograms between channel 36 (the green dot in Figure 1a) and the two segments before and after the excavation. The measured arrival time shift in Figure 4 only depends on the velocity within the boundaries of the DAS array. In Figures 4a and 4b, we observe that before construction started the surface wave arrivals show high spatial coherency in both segments. Their picked arrival times (red solid line) with slight moveout across the channels agree well with the computed arrival times (green dashed line) according to the average velocity of $816 \mathrm{~m} / \mathrm{s}$. Figures $4 \mathrm{c}$ and $4 \mathrm{~d}$ show the cross-correlograms two weeks after excavation on both segments. In Figure 4c, the south channels maintain the consistent arrival times at the reference velocity, indicating a stable subsurface environment between the two investigations (as expected because no excavation was performed along this ray path). In Figure 4d, systematic time delays are observed on the north segment, which suggests that the subsurface velocity between the two segments was reduced due to excavation of the basement.

To investigate the spatial sensitivity of the passive DAS monitoring system, we extract surface wave velocities from channels 162-205, where the construction site is between channel 172 and 184 . We use 3 channels near channel 205 as virtual sources to calculate normalized cross-correlations. Figure $5 \mathrm{a}$ and $5 \mathrm{~b}$ show one of the cross-correlograms (channel 205 as virtual source) before and after the excavation, respectively. The black lines denotes the picked travel time. Figure 5c displays the picked travel time versus distance (green dots: before excavation, red triangles: after excavation). The picked travel times stay within the same clusters with similar linear trends at channels east and west of the basement. At the basement, however, the cluster of red triangles deviates from that of the green circles, indicating significant changes in velocity.

The yellow dashed and blue solid lines are the least-squares piecewise linear fit of the red triangles and green dots of the three parts. As expected, at both the west (chan- 
(a)

2016/10/12
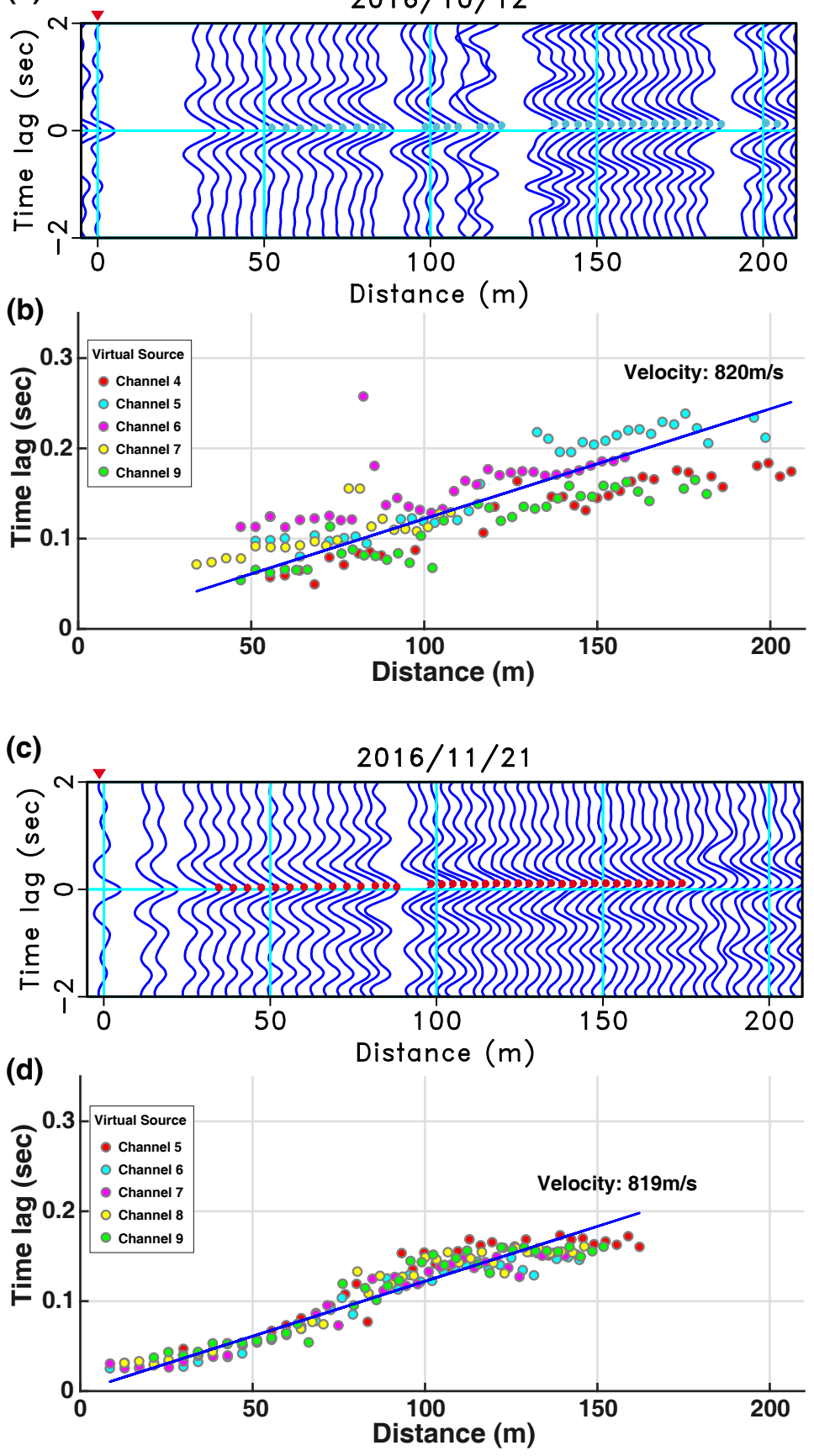

Figure 3. Five channels close to the channel 5 are used as virtual sources to calculate normalized cross-correlograms with channels 5-48. Plot (a) shows the normalized cross-correlograms on 12 October 2016. The channel used as virtual source (channel 5) is labeled with red triangle. The $\mathrm{x}$ axis denote the distance between virtual source and each receiver. Cyan dots denote the picked travel time lag. Plot (b) shows the picked time lag along the actual spatial distances from all the normalized cross-correlograms. The dots with different color denote the time delay picked with different virtual sources. Plot (c) and (d) are similar to (a) and (b), but computed on 21 November 2016. The marked surface seismic velocities are calculated by the slopes of the blue lines. 

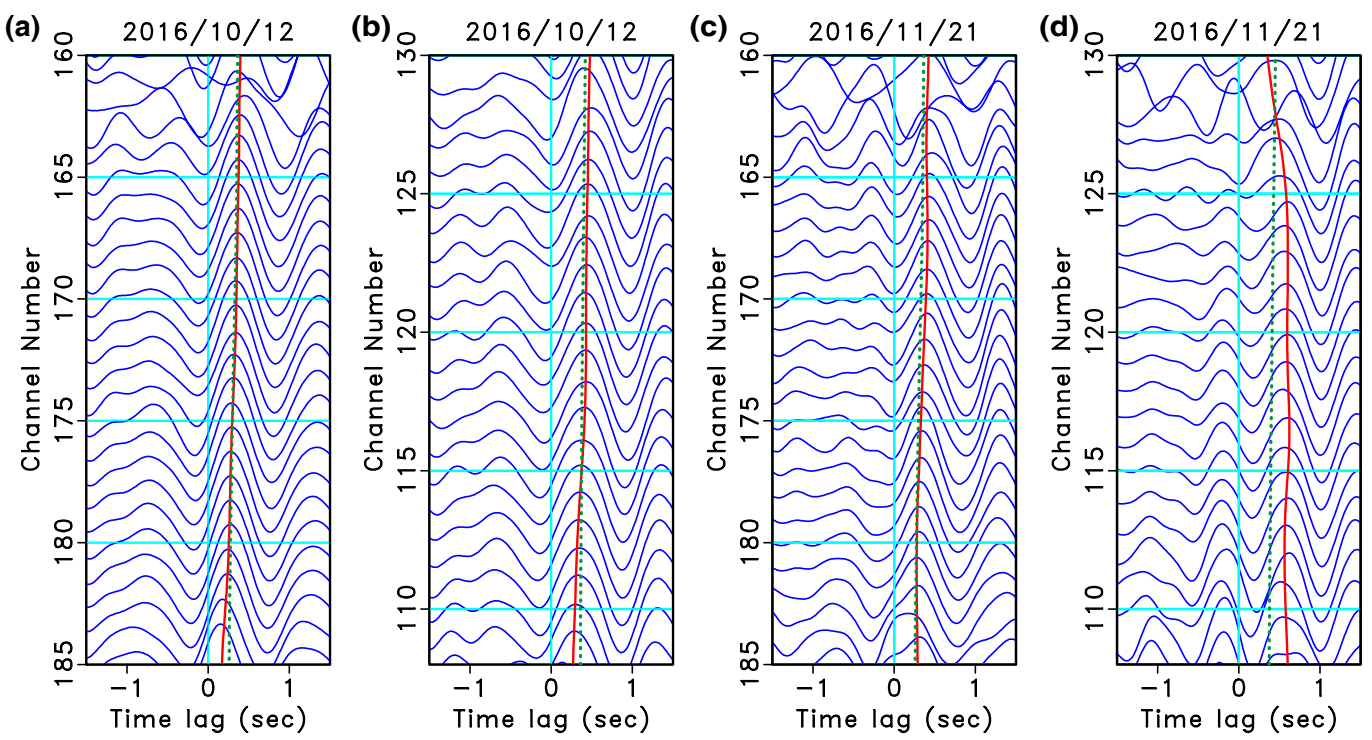

Figure 4. Plot (a) and (b) compare the normalized cross-correlograms between the virtual source (channel 36) and the front channels 165-183 and the back channels 108-136 on 12 October 2016, respectively. The virtual source location is denoted by the green dot in Figure 1a. The front and back channels are denoted by the orange and green lines in Figure 1a. Plot (c) and (d) are similar to plots (a) and (b), but on 21 November 2016. The green dashed lines show the calculated time lag according to the reference velocity, $816 \mathrm{~m} / \mathrm{s}$. The red solid lines show the picked time lag of the normalized cross-correlation.

nels 162-172) and east (channels 184-205) of the basement, the yellow and the blue lines have very similar slopes. However, across the basement (channels 172-184) the yellow line has a larger slope compared to the blue line, which indicates a lower subsurface velocity. Figure 5d displays the estimated average velocities along the three segments. Comparing the velocities before and after excavation, it is obvious that the velocities to the west and east of the basement are not significantly changed, whereas an apparent velocity drop from $824 \mathrm{~m} / \mathrm{s}$ to $721 \mathrm{~m} / \mathrm{s}$ is observed at the basement. The relative velocity drop is $12.5 \%$, nearly 4 times larger than the coefficient of variation $3.2 \%$ observed at stable sections of the array. Therefore, we believe the velocity drop is statistically significant, and caused by the excavation. This demonstrates the ability to detect changes due to the excavation of a single building basement with unprecedented resolution for a DAS-based urban seismic monitoring system.

\section{Discussion}

\subsection{Observed velocity variations by DAS}

Any monitoring system must strike an important balance between its sensitivity in detecting changes and its accuracy in issuing an alarm. In this study, we show that the velocity measured using a DAS array does vary in time and space. Factors leading to the velocity variations are three-fold: random DAS measurement error, changes in noise fields and source wavelet, and changes in subsurface geological conditions. Through careful signal processing, we have reduced the effect of DAS measurement noise, changes in noise fields and source wavelet, so as to improve our ability to isolate changes due to subsurface geological conditions. 

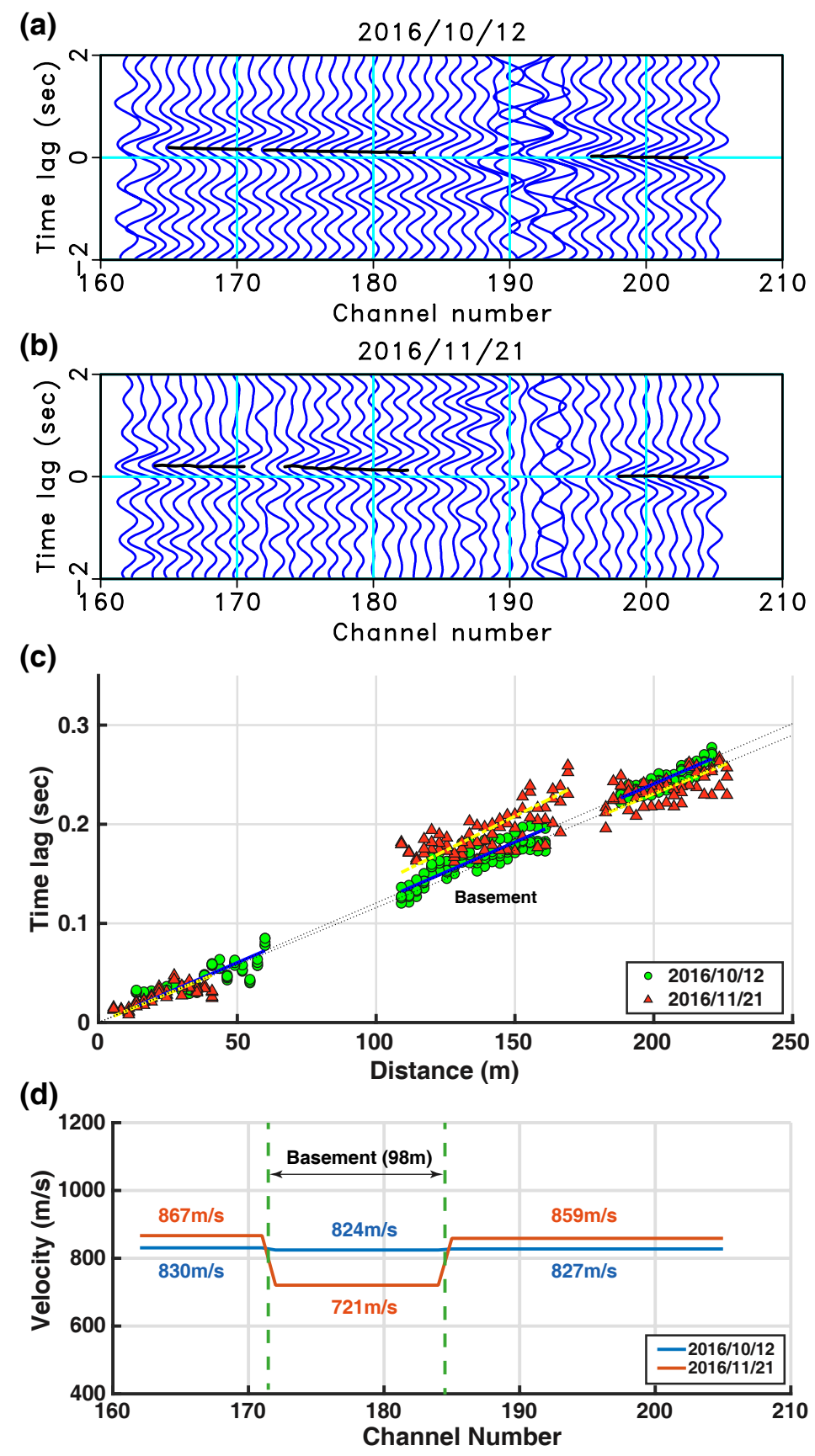

Figure 5. (a) and (b): Normalized cross-correlograms on 12 October 2016 and 21 November 2016, respectively. The black lines denote the picked travel time for three segments along the fiber cable. (c) Picked time lags on 12 October 2016 (Green circles) and 21 November 2016 (red triangles) plotted against distance. The gap from 60-100 meters distance are caused by removing the poor quality data around channel 193. The yellow dashed lines and the blue solid lines are least-squares linear fits to the red triangles and the green circles respectively. (d) Average velocities measured in three segments before and after excavation with a channel interval of $8.16 \mathrm{~m}$. 
Individual DAS channels have a lower signal-to-noise ratio (SNR) compared to conventional geophones in an ideal coupling condition (Lindsey et al., 2017; Yu et al., 2019). In this experiment, the fiber cable is loosely lying in an existing conduit, which further reduces the SNR. Moreover, DAS recordings in urban areas are severely contaminated by nearby construction and traffic noise. We observe a significant decrease in SNR after construction began, which reduces the sensitivity of velocity anomaly monitoring. The variations in measured velocity are quantified using data recorded in a geologically stable zone, and later used as baseline statistics to identify abnormal velocity variations caused by changes in subsurface geology.

With the Stanford DAS array, the measured velocity variation (12.5\%) after excavation provides strong statistical confidence of detection of an anomaly. On the other hand, the $3.2 \%$ baseline variance suggests that small changes in subsurface velocity may not be identified by the Stanford DAS system, which may limit its applicability to identify the development of small cavities in urban environments. With newer DAS interrogators with smaller channel spacing and gauge length and higher frequency noise sources, there is in principle a chance to detect smaller velocity changes, such as sinkhole development. These baseline statistics and sensitivities vary with site conditions, acquisition parameters, and signal characteristics. Establishing baseline velocity measurements and uncertainty bounds is very important for quantitative urban monitoring.

\subsection{Temporal resolution of DAS urban monitoring}

Many of the passive seismic methods assume far-field and full azimuthal random sources with equipartitioning in energy (Roberts \& Asten, 2008; Shapiro et al., 2005). However, urban ambient noise usually comes from fixed-location human activities that are often not perfectly random and isotropic (Bonnefoy-Claudet et al., 2006). When the sources are in close proximity to the array, longer recordings are used to increase the randomness and azimuthal coverage and reduce their susceptibility to near-field effects, particularly for the low SNR DAS recordings.

In this experiment, we make use of the repetitive quarry blasts as far-field, unidirectional sources to extract subsurface velocity based on much shorter recordings than would be required for an ambient noise approach. The temporal resolution of our experiment depends on the interval of the blasts, a few days in this case, which is sufficient for urban subsurface monitoring and alert. When an array is placed closer to a blast site that emits strong impulsive noises, abundant high-frequency signals may be recorded by DAS for higher spatial resolution subsurface monitoring.

\section{Conclusions}

Analysis of quarry blasts recorded by the Stanford Fiber Optic Seismic Observatory suggests that a surface DAS array in an existing communication infrastructure can be used for time-lapse monitoring of near-surface velocity changes. Compared to a $3.2 \%$ baseline velocity variation, a strong velocity decrease (12.5\%) is observed after two weeks of a basement excavation. The high temporal resolution is achieved by making use of repetitive quarry blast signals and a careful data processing workflow to remove the nearfield noise and to normalize the variations in the blasting conditions. Our study suggests that a DAS array deployed in existing communication infrastructure has a strong potential for high-resolution urban near-surface monitoring and urban geohazard risk management.

\section{Acknowledgments}

We would like to thank Biondo Biondi for inspiring us with this study and providing the DAS data. We thank editor Gavin Hayes, Ariel Lellouch, and an anonymous reviewer 
for their helpful comments. The cross-correlated DAS data used in this analysis are available at GitHub (https://github.com/GeoGANGFANG/StanfordDASQuarryBlast). We acknowledge the EDB Petroleum Engineering Professorship and Cambridge Sensing Pte Ltd for financial support. Yunyue Elita Li is funded by MOE Tier-1 Grant R302-000-182-114. Gang Fang is supported by National Natural Science Foundation of China (41504109). We also thank the Madagascar open-source software.

\section{References}

Ajo-Franklin, J. B., Dou, S., Lindsey, N. J., Monga, I., Tracy, C., Robertson, M., ... others (2019). Distributed acoustic sensing using dark fiber for near-surface characterization and broadband seismic event detection. Scientific reports, 9(1), 1328.

Biondi, B., Martin, E., Cole, S., Karrenbach, M., \& Lindsey, N. (2017). Earthquakes analysis using data recorded by the stanford das array. 87 th Annual International Meeting, SEG, Expanded Abstracts, 2752-2756.

Bonnefoy-Claudet, S., Cotton, F., \& Bard, P.-Y. (2006). The nature of noise wavefield and its applications for site effects studies: A literature review. EarthScience Reviews, 79(3-4), 205-227.

Dahm, T., Heimann, S., \& Bialowons, W. (2011). A seismological study of shallow weak micro-earthquakes in the urban area of hamburg city, germany, and its possible relation to salt dissolution. Natural Hazards, 58(3), 1111-1134.

Dean, T., Cuny, T., \& Hartog, H. A. (2017). The effect of gauge length on axially incident p-waves measured using fibre optic distributed vibration sensing. Geophysical Prospecting, 65(1), 84-193.

Díaz, J., Ruiz, M., Sánchez-Pastor, P. S., \& Romero, P. (2017). Urban seismology: On the origin of earth vibrations within a city. Scientific reports, 7(1), 15296.

Dou, S., Lindsey, N., Wagner, A. M., Daley, T. M., Freifeld, B., Robertson, M., ... Ajo-Franklin, J. B. (2017). Distributed acoustic sensing for seismic monitoring of the near surface: A traffic-noise interferometry case study. Scientific reports, $7(1), 11620$.

Douglas, I. (2004). People induced geophysical risks and urban sustainability. Washington DC American Geophysical Union Geophysical Monograph Series, 150, 387-397.

Gutiérrez, F., Parise, M., De Waele, J., \& Jourde, H. (2014). A review on natural and human-induced geohazards and impacts in karst. Earth-Science Reviews, $138,61-88$.

Jousset, P., Reinsch, T., Ryberg, T., Blanck, H., Clarke, A., Aghayev, R., ... Krawczyk, C. M. (2018). Dynamic strain determination using fibre-optic cables allows imaging of seismological and structural features. Nature communications, 9(1), 2509.

Lindsey, N. J., Martin, E. R., Dreger, D. S., Freifeld, B., Cole, S., James, S. R., ... Ajo-Franklin, J. B. (2017). Fiber-optic network observations of earthquake wavefields. Geophysical Research Letters, 44(23), 11-792.

Martin, E. R., Huot, F., Ma, Y., Cieplicki, R., Cole, S., Karrenbach, M., \& Biondi, B. L. (2018). A seismic shift in scalable acquisition demands new processing: Fiber-optic seismic signal retrieval in urban areas with unsupervised learning for coherent noise removal. IEEE Signal Processing Magazine, 35(2), 31-40.

Parker, T., Shatalin, S., \& Farhadiroushan, M. (2014). Distributed acoustic sensinga new tool for seismic applications. first break, 32(2), 61-69.

Renalier, F., Jongmans, D., Campillo, M., \& Bard, P.-Y. (2010). Shear wave velocity imaging of the avignonet landslide (france) using ambient noise cross correlation. Journal of Geophysical Research: Earth Surface, 115(F3).

Roberts, J., \& Asten, M. (2008). A study of near source effects in array-based (spac) microtremor surveys. Geophysical Journal International, 174(1), 159-177. 
Schenato, L., Palmieri, L., Camporese, M., Bersan, S., Cola, S., Pasuto, A., ... Simonini, P. (2017). Distributed optical fibre sensing for early detection of shallow landslides triggering. Scientific reports, 7(1), 14686.

Shapiro, N. M., Campillo, M., Stehly, L., \& Ritzwoller, M. H. (2005). Highresolution surface-wave tomography from ambient seismic noise. Science, 307(5715), 1615-1618.

Spica, Z., Perton, M., Martin, E. R., Biondi, B., \& Beroza, G. (2019). Urban seismic site characterization by fiber-optic seismology. EarthArXiv.

Tran, K. T., \& Sperry, J. (2018). Application of 2d full-waveform tomography on land-streamer data for assessment of roadway subsidence. Geophysics, 83(3), EN1-EN11.

Willis, M. E., Barfoot, D., Ellmauthaler, A., Wu, X., Barrios, O., Erdemir, C., ... Quinn, D. (2016). Quantitative quality of distributed acoustic sensing vertical seismic profile data. The Leading Edge, 35(7), 605-609.

Yu, C., Zhan, Z., Lindsey, N. J., Ajo-Franklin, J. B., \& Robertson, M. (2019). The potential of das in teleseismic studies: Insights from the goldstone experiment. Geophysical Research Letters, 46(3), 1320-1328.

Zhang, Y., Li, Y. E., Zhang, H., \& Ku, T. (2019). Near-surface site investigation by seismic interferometry using urban traffic noise in singapore. Geophysics, 84(2), B169-B180. 